\title{
Insulin receptor substrate 2: a bridge between Hippo and AKT pathways
}

\author{
Sun-Hye Jeong ${ }^{*} \mathcal{E}$ Dae-Sik Lim \\ National Creative Research Initiatives Center, Department of Biological Sciences, Biomedical Research Center, Korea Advanced Institute of \\ Science and Technology (KAIST), Daejeon 34141, Korea
}

\begin{abstract}
NAFLD induces the development of advanced liver diseases such as NASH and liver cancer. Therefore, understanding the mechanism of NAFLD development is critical for its prevention and treatment. Ablation of PTEN or Hippo pathway components induces liver cancer in a murine model by hyperactive AKT or YAP/TAZ, respectively. Although the regulation of these two pathways occurs in the same hepatocyte, the details of crosstalk between Hippo-YAP/TAZ and PTEN-AKT pathways in liver homeostasis and tumorigenesis still remain unclear. Here, we found that depletion of both PTEN and SAV1 in liver promotes spontaneous NAFLD and liver cancer through hyperactive AKT via YAP/TAZmediated up-regulation of IRS2 transcription. Conversely, NAFLD is rescued by both ablation of YAP/TAZ and activation of the Hippo pathway. Furthermore, human HCC patients with NAFLD showed strong correlation between YAP/TAZ and IRS2 or phospho-AKT expression. Finally, the inhibition of AKT by MK-2206 treatment attenuates NAFLD development and tumorigenesis. Our findings indicate that Hippo pathway interacts with AKT signaling during the intervention with IRS2 to prevent NAFLD and liver cancer. [BMB Reports: Perspective 2018; 51(5): 209-210]
\end{abstract}

*Corresponding author. E-mail: sunhye8@kaist.ac.kr

https://doi.org/10.5483/BMBRep.2018.51.5.095

Received 10 April 2018

Keywords: AKT signaling, Hepatic steatosis, Hippo pathway, IRS2, Liver cancer

Abbreviations: DKO, Double knock-out; HCC, Hepatocellular carcinoma; IRS2, Insulin receptor substrate 2; NAFLD, Non-alcoholic fatty liver disease; NASH, Non-alcoholic steatohepatitis; PTEN, Phosphatase and tensin homolog; SAV1, Salvador homolog 1; TAZ, Transcriptional coactivator with PDZ-binding motif; YAP1, Yes-associated protein 1

Perspective to: Sun-Hye Jeong et al (2018) Hippo-mediated suppression of IRS2/AKT signaling prevents hepatic steatosis and liver cancer, The Journal of Clinical Investigation 3(95802) doi:10. 1172/JCI95802
NAFLD is frequently observed in severe obese population and progresses to advanced diseases such as $\mathrm{NASH}$, cirrhosis, and liver cancer. Approximately, 25.24\% of the global population is reported to manifest NAFLD, and $25 \%$ of patients diagnosed with NAFLD develop NASH (Younossi ZM et al (2016) Hepatology 64, 73-84). However, the molecular mechanism underlying the disease or its treatment is unknown (Chi KR (2015) Nat Rev Drug Discov 14, 447-448). Therefore, it is imperative to investigate the mechanism of NAFLD/NASH using an appropriate mouse model, which mimics human liver disease progression.

The Hippo pathway consists of serial kinases and scaffold proteins including MST1/2-SAV1 and LATS1/2-MOB1, which phosphorylate and inhibit the oncoproteins YAP and TAZ (Johnson R et al (2014) Nat Rev Drug Discov 13, 63-79). In case of Hippo inactivation, YAP/TAZ translocate from cytoplasm to the nucleus and bind to the transcriptional factors TEAD1/2/3/4 (Zanconato $F$ et al (2016) Cancer Cell 29, 783-803). In the murine model, liver-specific knock-out of Hippo components leads to liver tumorigenesis via YAP/TAZ hyperactivation (Zhou D et al (2009) Cancer Cell 16, 425-438 and Lee KP et al (2010) Proc Natl Acad Sci USA 107, 8248-8253). Nonetheless, the role of Hippo-YAP/TAZ in metabolic liver disease, which precedes tumorigenesis is not well-known. While a recent study revealed that diet-induced NASH mouse shows elevated TAZ expression, which induces Indian hedgehog (lhh) and activates stellate cells (Wang X et al (2016) Cell Metab 24, 848-862), the mechanism underlying the role of Hippo pathway in NAFLD and genetic modification-based evidence supporting the TAZ-Ihh axis in vivo are unknown.

AKT acts as a main hub linking several downstream components such as GSK3, TSC2/mTOR, FOXO, p21 and p27, and subsequently acts as a determining factor in tumorigenesis (Manning BD et al (2017) Cell 169, 381-405). In addition, AKT is associated with the development of NAFLD by regulating the lipogenic transcriptional factor SREBP1C (Krycer JR et al (2010) 21, 268-276), which is validated by the hydrodynamic injection of myristoylated AKT (constitutive active $A K T$ ) and liver-specific PTEN KO mice, resulting in NAFLD, NASH, and hepatocellular carcinoma (HCC) (Calvisi DF et al (2011) Gastroenterology 140, 1071-1083 and Horie Y 


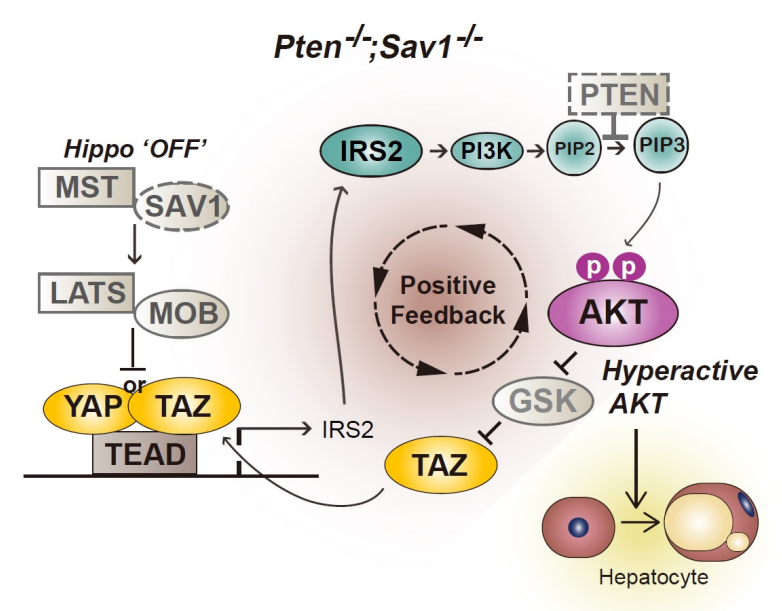

Diagram 1. Schematic diagram showing the positive feedback loop between Hippo-YAP/TAZ and IRS2-AKT signaling in the DKO livers. Hyperactive YAP/TAZ in the impaired Hippo pathway resulted in the induction of IRS2, leading to amplification of AKT activity in the absence of PTEN. The hepatocyte containing a hyperactive AKT promotes de novo lipogenesis and subsequent hepatic steatosis.

et al (2004) J Clin Invest 113, 1774-1783). It has been reported that YAP activates AKT by down-regulating PTEN via YAP-mediated induction of mir-29 and that Yap is linked to the AKT-Skp2-p27 axis in liver tumorigenesis (Tumaneng $\mathrm{K}$ et al (2012) Nat Cell Biol 14, 1322-1329; and Zhang S et al (2017) Cancer Cell 31, 669-684). However, Jeong et al. refutes previous studies by reporting the absence of any differences in pAKT expression between WT and Sav $1^{-1-}$ liver (hyperactive YAP condition) as well as differences in PTEN expression between WT and $\mathrm{Yap}^{-1-} \mathrm{Taz}^{-1-}$ mice. Therefore, a more precise mechanism of the crosstalk between Hippo and AKT signaling needs to be elucidated.

Here, we generated liver-specific PTEN and SAV1 mice (Pten ${ }^{-1-} \mathrm{Sav}^{-1-}$ or DKO) to investigate the interaction between the Hippo and AKT pathways in vivo (Diagram 1). DKO mice displayed spontaneous progression of NAFLD, NASH, and cirrhosis leading to cancer, in which each step progresses much faster than Pten ${ }^{-1-}$ livers. Hyperactivion of AKT in livers of DKO mice was the major trigger for NAFLD development. AKT inhibition via the clinical trial phase II AKT inhibitor (MK-2206) attenuated the development of NAFLD and liver tumorigenesis in DKO mice. Collectively, the findings suggest that the interaction between Hippo and AKT signaling is important for suppression of hepatic steatosis by inhibiting AKT activity.

Using several mouse models and in vitro approaches, we revealed that YAP/TAZ hyperactivation potentiates AKT activity via transcriptional induction of IRS2, the upstream component of AKT. YAP/TAZ and TEAD bind to the early promoter and intron regions of $I r s 2$, and activate the transcription of Irs2. Up-regulated IRS2 enhances PI3K activity and amplifies the activity of AKT in the absence of PTEN. This result was confirmed by generating $\operatorname{Pten}^{-1-} \mathrm{Sav}^{-1-} \mathrm{Yap}^{-1-} \mathrm{Taz}^{-1-}$ mice, in which the liver was rescued from NAFLD compared with DKO mice. In addition, for activating the Hippo pathway, we generated MST1 (Hippo, a binding partner of SAV1) transgenic mouse to see whether the active Hippo pathway has similar effect with YAP/TAZ deletion. We also found a reduced NAFLD phenotype through a decrease in YAP/TAZ activity. Abrogation of IRS2 by AAV-CRISPR-Cas9 in DKO mice ameliorated the progression of liver cancer via decreased pAKT activity. To further confirm our results in humans, we found that the expression of TAZ, YAP, IRS2, and pAKT was higher in HCC patients developed from NAFLD compared with those without NAFLD. Overall, we have identified the positive feedback loop connecting Hippo-YAP/TAZ with IRS2-AKT in the development of NAFLD, which provides novel insight into the treatment of liver diseases. Furthermore, since DKO mice develop NAFLD and liver cancer that reflect the progression of human liver disease, the DKO mouse represents a valuable model for screening of drugs targeting AKT.

\section{ACKNOWLEDGEMENTS}

This study was supported by a grant awarded by the National Creative Research Initiatives Grant (NRF-2010-0018277, to D.-S.L.), the Korea Mouse Phenotyping Project (NRF-2014 M3A9D5A01075128, to S.-H.J.) and the TJ Park Science Fellowship of POSCO TJ Park Foundation (to S.-H.J.). 